\title{
Una primera cronografía de la vida cotidiana del adulto mayor en los Alpes. Nuevas herramientas para favorecer la adaptación
}

\author{
LUC GWIAZDZINSKI \\ > UMR Pacte CNRS 5194, Université Grenoble Alpes, Suiza. Iuc.gwiazdzinski@gmail.com \\ FLORENT CHOLAT \\ > UMR Pacte CNRS 5194, Université Grenoble Alpes, Suiza. florent.cholat@gmail.com \\ Universidad de Valparaíso \\ Facultad de Arquitectura \\ Revista Márgenes \\ Espacio Arte Sociedad \\ Una primera cronografía de la vida cotidiana del adulto mayor en \\ los Alpes. Nuevas herramientas para favorecer la adaptación \\ Diciembre 2016 Vol $13 \mathrm{~N}^{\circ} 19$ \\ Páginas 32 a 37 \\ ISSN elec. 0719-4463 \\ ISSN imp. 0718-4034 \\ Recepción: agosto 2016 \\ Aceptación: noviembre 2016
}

\section{RESUMEN}

Cuando se toma el tiempo de conversar con un adulto mayor, nos obligan a cambiar la mirada sobre ellos e invitan a imaginar con ellos soluciones concordantes a sus necesidades y anhelos: Ya no queremos que nos consideren enfermos sino como verdaderos ciudadanos; No porque tengamos la misma edad, tenemos los mismos gustos o personalidad. Estos discursos, ponen en evidencia la importancia del territorio y de los servicios cotidianos en la calidad de vida: Si pudiéramos tener un buen sistema de transporte para desplazarnos, tendríamos más libertad de movimiento, sería más que suficiente. Integran la cuestión del envejecimiento en un contexto más global, incorporando la ciudad y la urbanidad: el derecho a la ciudad para todos aquí y ahora, independientemente de la edad. Agregan que Mientras pueda quedarme aquí en mi barrio, me quedaré. Se tomó ese tiempo de ir al encuentro de los adultos mayores para conocer su realidad.

PALABRAS CLAVE

envejecimiento, vida cotidiana del adulto mayor, calidad de vida

A first chronology of seniors daily life in the Alps. New tools to favor adaptation ABSTRACT

When one takes time to talk with a senior, we are forced to change our perspective about them and they invite to imagine together practical solutions for their needs and wants: We don't want to be considered as sick but as true citizens; Just because we're all the same age doesn't mean we have the same interests or personality. These speeches make evidence for the importance of territory and daily services in life quality: if we could have a good transportation service, we'd have more freedom of movement, it would be more than enough. They integrate the subject of aging in a global context, including the city and urban themes: the right to the city for everyone right here, right now, independent of their age. They add As long as I can stay in my neighborhood, I'll stay. The time to go and share with seniors to understand their reality was taken.

KEYWORDS

agging, daily life of seniors, life quality

INTRODUCCIÓN

De la cama a la ventana, de la cama al sillón, luego de la cama a la cama.

Jacques Brel, 1963

Cuando se toma el tiempo de conversar con un adulto mayor, nos obligan a cambiar la mirada sobre ellos e invitan a imaginar con ellos soluciones concordantes a sus necesidades y anhelos: Ya no queremos que nos consideren enfermos sino como verdaderos ciudadanos; No porque tengamos la misma edad, tenemos los mismos gustos o personalidad. Estos discursos, ponen en evidencia la importancia del territorio y de los servicios cotidianos en la calidad de vida: si pudiéramos tener un buen sistema de transporte para desplazarnos, tendríamos más libertad de movimiento, sería más que suficiente. Integran la cuestión del envejecimiento en un contexto más global, incorporando la ciudad y la urbanidad: el derecho a la ciudad para todos aquí y ahora, independientemente de la edad. Agregan que Mientras pueda quedarme aquí en mi barrio, me quedaré. Se tomó ese tiempo de ir al encuentro de los adultos mayores para conocer su realidad. 


\section{LOS DESAFÍOS EN EL ENVEJECIMIENTO}

Para envejecer y seguir con buena salud, el adulto mayor necesita de actividad física y cuidados; por otro lado, las relaciones les permite participar de la vida social y limita los riesgos asociados a la soledad como la depresión, que se manifiesta en un cuadro de angustia (Chattat, 2004), ver las psicopatologías (Fontaine, 2007). En un contexto de pérdida de autonomía, la capacidad de las personas mayores para desplazarse y así socializar, son tan importantes como la ayuda de algún familiar, amigo e incluso los servicios comerciales. Nuestros mayores están cada vez más solos, es lo que recalca la Fundación de Francia en su informe sobre "Las soledades en Francia" (Fondation de France, 2014), en donde señala que el $27 \%$ de las personas de 75 años y más, sufrirían de aislamiento, en resumen, mientras más se vive, menor es la posibilidad de encontrarse con personas de la misma edad.

Las personas mayores pierden sus seres próximos y en algunos casos, se enfrentan a la viudez, compleja situación que les obliga a reorganizar y adaptarse a nuevos modos de vida (Caradec, 2001). A pesar de que la pérdida de autonomía, no necesariamente está ligada al envejecimiento, sí es un factor de aislamiento; en Francia 1,15 millones de franceses son dependientes (INSEE, 2012), la mayoría debido a su avanzada edad. La dependencia refiere a dificultades para hablar, vestirse, alimentarse o desplazarse, en otras palabras, implica no poder realizar solos tareas cotidianas. Para hacer frente a dicha situación que limita el encuentro con el otro, las sociedades se han organizado mediante el apoyo y cuidados necesarios para estas personas.

Junto a la pérdida de autonomía, el individuo, adapta su modo de vida para luchar contra la soledad y el aislamiento puesto que la interacción con el otro, ya sea directa o a través de los medios de comunicación, es necesaria a una buena calidad de vida. De esta forma, para la persona aislada, una primera estrategia consiste en suplir el contacto directo (de persona a persona) a otras formas de relaciones sociales, indirectas, como el teléfono, el correo e incluso más recientemente internet.

Si bien, el teléfono es un simple intercambio vocal con la red social, se observa que existe una correlación entre la cantidad de contactos directos e indirectos, en particular aquellos relativos a la familia (Segalen, 1999). No obstante, las personas no compensan la cantidad de visitas por la cantidad de contactos telefónicos, de hecho, los individuos tienden a mantener contactos indirectos independientemente de sus contactos directos. Dichas prácticas varían en función del género, por ejemplo las mujeres sostienen intercambios telefónicos bastante más largos que los hombres, del mismo modo, la relación telefónica madre/hija es más extensa (Segalen, 1999).

El correo es utilizado por algunos para intercambiar cartas e internet permite el encontrar nuevos contactos virtuales, sin embargo, ambas son prácticas secundarias en comparación al teléfono, el primero está en declive y el segundo está aún poco extendido en la población adulto mayor.

De este modo, la televisión se transforma en una compañía para las personas mayores. Además de un analizador del envejecimiento (Vincent Caradec, 2003), es un lugar de dialógo consigo mismo, (Berger, Luckmann, 1986), en efecto, contribuye a mantener conversaciones internas para paliar la falta de contacto, es la devoradora del tiempo libre de los ancianos (Paillat, 1993). Al contrario, el uso de la radio es más breve, pese a que es apreciado por aquellas personas con problemas de visión.
Para las personas de más edad, la prensa escrita sigue siendo importante, el soporte más leído es la prensa regional que relata información local, permitiéndole mantenerse informada sobre la vida que ocurre en su territorio. En segundo lugar, se encuentra la prensa semanal o mensual, tipo revista con información de otros lugares, mediante el cual, los adultos mayores se evaden y cambian de contexto. (Caradec, 2003). Es así que los mayores viven su territorio y lo representan a través de las herramientas de comunicación y los medios (Viriot-Durandal et al., 2012).

Un indicio de la importancia de los medios de comunicación en la vida de las personas mayores en la etapa cercana a la muerte, que se manifiesta con el ralentizamiento general, el decrecimiento de la impulsión vital, la tendencia a retraerse; no sólo se mide por la disminución de las relaciones sociales de la persona (Cumming, Henry, 1961), sino también por el menor uso de la televisión, el abandono de la lectura y la baja de los contactos telefónicos. En consecuencia, resulta primordial, observar el contexto psico-social en el estudio del envejecimiento.

\section{LA ELECCIÓN DEL CUIDADO A DOMICILIO}

Una de las formas de responder a la situación expuesta anteriormente, es favorecer el cuidado de las personas en su domicilio, apelando a la sociedad en su conjunto, dado el aumento de la población adulto mayor y sus efectos en el cambio de la estructura familiar. Asimismo, es un reto financiero, más aún si se propone como alternativa a la internación en costosas instituciones que no dan a basto con la demanda, por otro lado, es una forma de mejorar la calidad de vida, evitando la ruptura familiar y su consiguiente pérdida de referentes; finalmente, es una prueba de ciudadanía para el adulto mayor dándole la posibilidad de seguir participando en la vida local.

Esta modalidad, menos costosa y más humana que las instituciones (casa de retiro, establecimientos de salud, etc.) será inevitablemente una alternativa en vista del aumento de las personas de edad en situación de dependencia, es decir que la elección de la modalidad, tiene repercusiones en la vida cotidiana del adulto mayor y para la sociedad en su conjunto. Por ejemplo, algunas personas no hablan con nadie durante un período de 4 días, otras viven con menos de 300 euros al mes, por lo demás, viven en condiciones poco salubres. Sin embargo, el modelo de mantención y cuidado en domicilio requiere de un conjunto de movilizaciones que está sujeta a una red de apoyo, familiares o de profesionales, entrando en contradicción con los principios del desarrollo sostenible.

De esta forma, formulamos la hipótesis de que la permanencia y cuidado del adulto mayor en domicilio conlleva un riesgo de aislamiento para las personas en situación de dependencia y que requiere de un conjunto de movilizaciones asociadas, no obstante, dichas movilizaciones son también un recurso social que participa de la habitabilidad de estos espacios.

De este modo, el objetivo de la esta investigación apunta a levantar primero una crono-geografía exploratoria y performativa con el fin de estudiar los impactos sociales y territoriales de los efectos de la pérdida de autonomía derivados del envejecimiento. Se propone observar el tiempo del adulto mayor entrevistado junto a la movilización inversa ${ }^{1}$ producto de la pérdida de autonomía. Para ello, se levantó datos de carácter cualitativo a través de entrevistas semi estructuradas y cuantitativos mediante la confección de una base de datos y uso de GPS, en dos territorios: la zona francesa e italiana de los Alpes. Se pudo medir el tiempo y movilidad de un grupo 


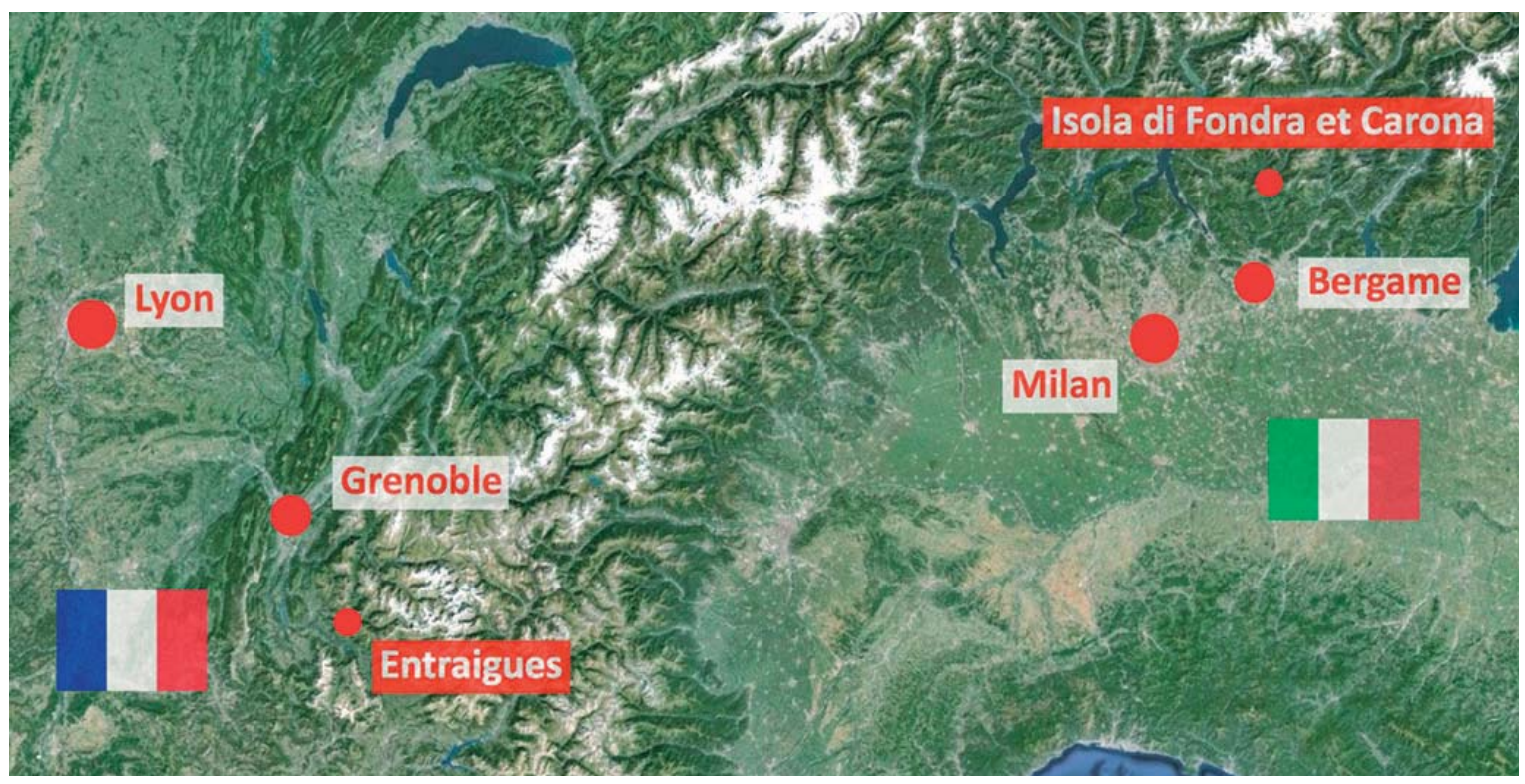

de individuos, principalmente de mujeres mayores en situación de dependencia que ya hayan pasado la etapa de viudez.

Para estudiar las repercusiones derivadas de situación de dependencia sobre el tiempo y movilidad del adulto mayor, se eligió usar el índice $\mathrm{AGGIR}^{2}$ (autonomía gerontológica de los grupos Iso-recursos) además de elegir una muestra compuesta por individuos provenientes de las categorías GIR 6, GIR 5 y GIR 3 por las siguientes razones:

- La categoría GIR 6, presenta un particular interés, en efecto, se puede observar la realidad de individuos relativamente autónomos, es decir, sólo requieren de apoyo (ADMR ${ }^{3}$ en Francia) en forma puntual para la realización de tareas domésticas y sociales (ilustrativas), a pesar de que dicha clasificación no siempre corresponde totalmente a la situación real.

- La categoría GIR 5 también es importante, en esta categoría, los adultos mayores requieren de apoyo regularmente para la realización de actos no discriminatorios (ilustrativos) de la vida cotidiana. En general, son personas que presentan dificultades para ejecutar actividades diarias, siendo el inicio de una situación de dependencia que requiere de apoyo.

- La categoría GIR 3 se destaca de las anteriores puesto que alude a una pérdida de autonomía importante, es así que las personas necesitan de ayuda varias veces durante el día para la realización de actos discriminatorios cotidianos

El análisis detallado de los entrevistados y entrevistadas nos permite proponer formatos ilustrativos innovadores a partir de datos habitualmente no tratados; se presentarán tres formas de ilustrar el fenómeno estudiado particularmente pertinente en este contexto.

Una primera propuesta consiste en representar el conjunto de los contactos sociales de una persona a través de un histograma con el fin de clasificarlos de acuerdo al tiempo de intercambio con el individuo entrevistado. Esta representación no hace la distinción entre los individuos pero se puede visualizar el tipo de intercambio, es decir si este es directo o indirecto (correo, teléfono o internet).

En el gráfico, se observa que el tiempo relacional varía bastante de un individuo a otro, así como la cantidad de personas con quienes

34 > Revista Márgenes № 19 Vol 13 > Diciembre 2016: 32 a 37 Facultad de Arquitectura > Universidad de Valparaíso
> ISSN electrónico 0719-4436 $>$ ISSN impreso 0718-4034 


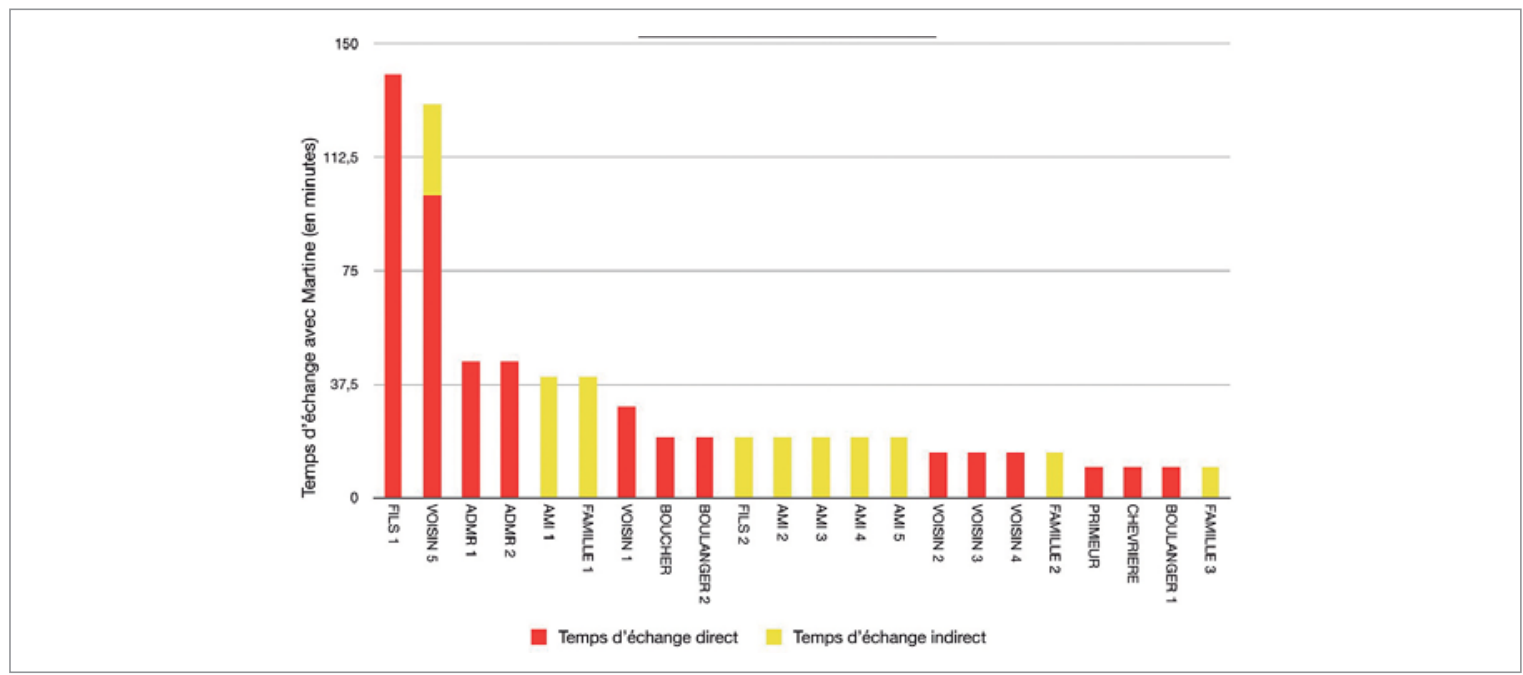

ción de alta dependencia y el aumento de la cantidad de personas en contacto con el adulto mayor, es decir persona externa que lo asiste; en contraste, dicha tendencia es inversa en Italia.

La segunda representación gráfica, se denomina "crono-geografía" corresponde a una geografía virtual de los contactos sociales del adulto mayor estudiado (en el centro de este gráfico). Cada círculo representa una persona con la que se relaciona, estos se han organizados en 4 categorías (familia, amigos, servicios comerciales y servicios médico-sociales). Mientras mayor es el diámetro del círculo, mayor es el tiempo de interacción, por otro lado si estos se encuentran más cerca del centro, mayor es la frecuencia de contacto.

El gráfico, muestra la importancia del tiempo de interacción, por ejemplo, entre el individuo estudiado y su apoyo, de esta manera, es posible analizar la importancia de la frecuencia y del tiempo de intercambio en la relación que une a ambas personas.

En cada zona de los Alpes, se observa que el entorno social de los adultos mayores varía mucho entre los diferentes entrevistados, la dimensión temporal revela tres hechos acerca de la vida de estos:

- $\quad$ Existe similitudes en relación al tiempo de apoyo a un mismo grado de dependencia.

- Se distinguen tres grupos dominantes en la vida de la persona mayor, amigos, familia y servicios médico-sociales en los tres tipos de grado de dependencia estudiado.

- La diferencia en la forma de intervención de las personas en situación de dependencia: apoyo desde el domicilio en el caso de Italia y apoyo externo en Francia.

- $\quad$ Las políticas públicas se dividen en distintos tipos de ayuda para la persona mayor en su domicilio:

- $\quad$ El apoyo medicinal que realizan las enfermeras, médicos u otro cuya finalidad es el cuidado y el seguimiento de todas las patologías que pudiesen desarrollar cada persona.

- $\quad$ El apoyo directo a la persona, realizado por paramédicos que se centra en la ayuda de tareas cotidianas como comer, ayuda en la limpieza personal, desplazamientos en el domicilio o externo, acompañamiento durante la noche.

- El apoyo en tareas domésticas a cargo de un aseador(a) que realiza la limpieza, el planchado o la elaboración de comidas por ejemplo.

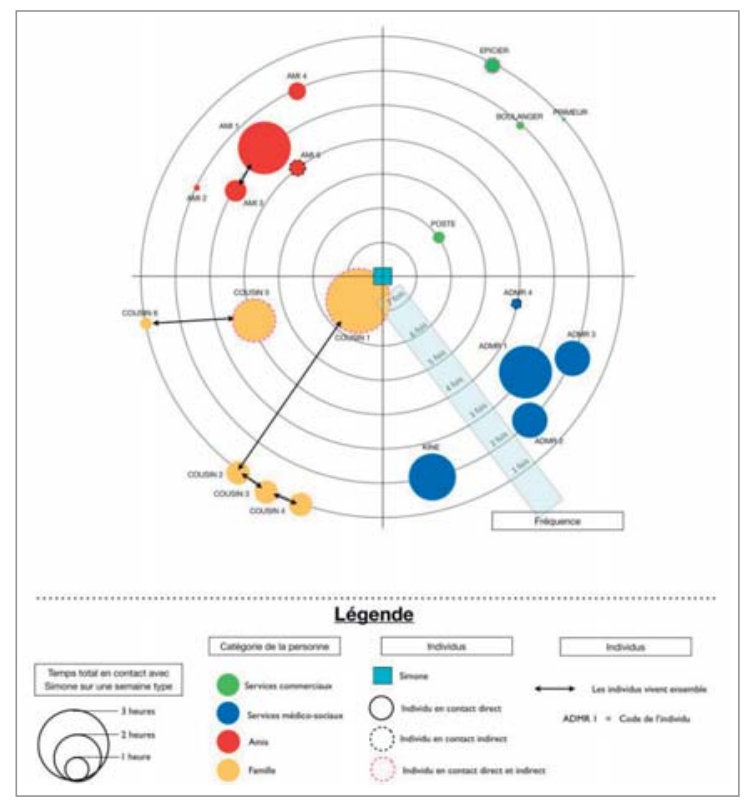

$>$ Gráfico1. Balance relacional de Martine.

$>$ Gráfico 2. Crono-geografía de intercambios sociales de Simone. Fuente Florent Cholat, 2013. 


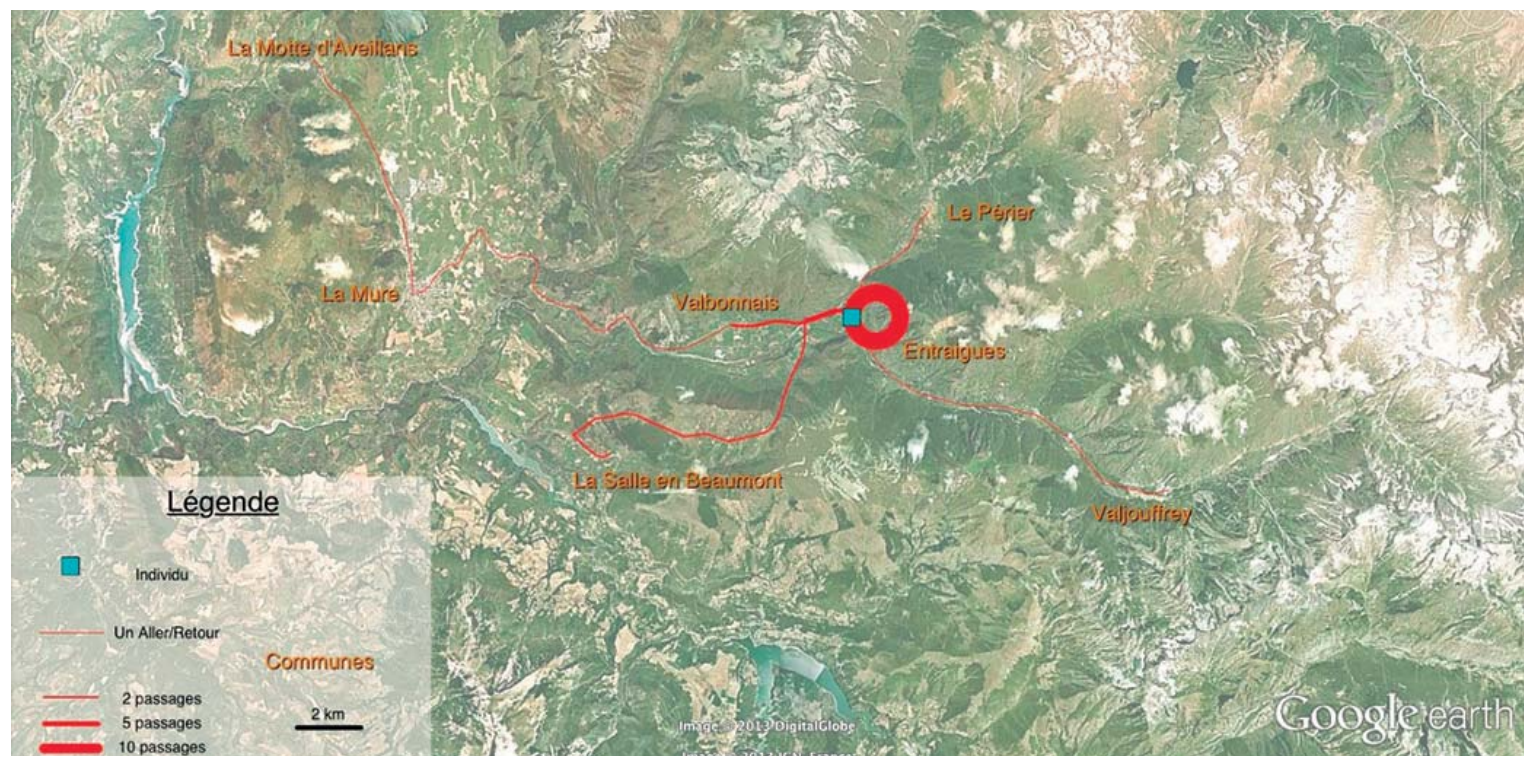

- El apoyo en los desplazamientos externos que permite mantener la movilidad de las personas para asistir al médico, ir de compras o actividades de ocio.

- El apoyo en las relaciones externas, apoyo en la administración, consejos y otro tipo de asistencia por ejemplo en diferentes trámites.

- Apoyo complementario para la realización de pequeños trabajos y tareas extraordinarias. (Fuente: Ministerio de desarrollo social y de la salud, Francia ${ }^{4}$ )

Por otro lado, junto con los diferentes apoyos a domicilio, existe una importante cantidad de personas que mantienen contacto con las personas en situación de dependencia en el mismo territorio. En la cartografía siguiente, el cuadrado celeste, representa el domicilio de la persona, las líneas rojas su movilidad. El grosor de dicha línea aumenta cada vez que se vuelve a pasar por un mismo camino. Las marcas circulares que parten y terminan en el domicilio de la persona, refieren a la movilidad dentro del pueblo.

Si se observan las diferentes cartografías, lo primero que se destaca en este territorio de los Alpes, es que evidentemente, la movilidad se estructura a lo largo del sistema de los valles, por lo demás, se constata que tanto en Francia como en Italia, se produce un cruzamiento de las escalas.

En primer lugar, las escalas comerciales, en Italia, gran parte del comercio se encuentra cerca del domicilio, permitiendo un aprovisionamiento a escala local, situación inversa en Francia. Dicho de otro modo, en Italia el pequeño comercio es un servicio utilizado por el adulto mayor mientras que en Francia, este servicio de comercio es itinerante, siendo el comerciante que ofrece sus productos a domicilio.

En el estudio que se realizó acerca de la movilidad producto de la situación de dependencia del adulto mayor, se puede avanzar que, en la medida que pierde su autonomía, menos moviliza/utiliza los recursos dentro del pueblo. Situación que se explica por la pérdida de autonomía en el desplazamiento y la distancia con los servicios médico-sociales y de las relaciones familiares.

> Figura 2. Mapa (falta descripción). Fuente: Florent Cholat, junio 2013.

Finalmente, basándose en la cantidad de kilómetros recorridos por las personas entrevistadas y el cruzamiento de escala, se advierte

36 > Revista Márgenes № 19 Vol 13 > Diciembre 2016: 32 a 37 Facultad de Arquitectura > Universidad de Valparaíso 
que la situación de dependencia en Italia disminuye la movilidad inversa ${ }^{5}$, sin embargo, dicho fenómeno no es tan notorio en Francia debido a los numerosos apoyos externos que recibe el adulto mayor.

En un contexto de rigurosidad presupuestaria en donde el financiamiento público es cada vez más complejo, es importante considerar el costo de las políticas de apoyo a domicilio, en efecto, de todas las personas que colaboran con el adulto mayor, sólo los servicios de apoyo a domicilio son remunerados ya sea directamente por el (beneficiado por un subsidio), por el estado o la caja de jubilados.

La pregunta es, cuál sería el costo real de dicho servicio en relación al conjunto de las personas que participan de la vida del adulto mayor, luego de confeccionar un balance económico de la semana tipo descrita en este estudio, se propone, calcular el monto real del servicio tomando en cuenta el costo por hora de las diferentes profesiones involucradas. Se observa, que el costo del apoyo aumenta en relación al grado de dependencia, es así, que en el caso de dependencia importante, el costo es elevado en ambos casos (a domicilio o en institución). Para terminar, se llegó a la constatación que el costo de una persona en un establecimiento es de $1000 €$ por semana que varía según la estructura de este, mientras que el costo del apoyo a domicilio es levemente más elevado.

El problema del costo derivado la situación de dependencia del adulto mayor, podría plantearse desde otro ángulo, en efecto, desde un punto de vista estrictamente económico, los montos descritos son parte de una suerte de economía local. Estos montos aportados por diferentes organismos son percibidos por la localidad y por las personas que proporcionan el servicio de apoyo, de esta manera, se podría considerar que el territorio se beneficia económicamente de dicha situación. Asimismo, ocurre con el comercio itinerante en la medida que presta servicio a las personas que perdieron su movilidad, manteniéndose a domicilio.

NOTAS

1 Movilidad inversa refiere al conjunto de personas que se movilizan entorno al adulto mayor, limitando su propia movilización/ desplazamiento.

2 El AGGIR mide el grado de dependencia del adulto mayor que postula a un subsidio personalizado de autonomía (se mide del 1 al 6, siendo el 1 el mayor grado de dependencia), el índice evalúa la capacidad del adulto mayor para realizar 10 actividades corporales y mentales (discriminatorias) además de 7 actividades domésticas y sociales (ilustrativas). Sólo las primeras 10 actividades son utilizadas para definir el GIR, grado de dependencia; las actividades ilustrativas son complementarias.

3 ADMR, Fundado en 1945, en su origen fue "Aide á domicile en milieu rural" que es ayuda en domicilio en medio rural, se convirtió en una red de asociaciones que ofrece apoyo a domicilio (tareas domésticas, acompañamiento en los desplazamientos) en el conjunto del territorio destinados a diferentes grupos entre los cuales al adulto mayor.

4 Ministère des affaires sociales et de la santé, France.

5 Movilidad inversa refiere al conjunto de personas que se movilizan entorno al adulto mayor, limitando su propia movilización / desplazamiento, por ejemplo en el caso de Italia, las personas se aprovisionan en el comercio local.

\section{BIBLIOGRAFÍA}

BERGER, P. et T. LUCKMANN (1986) La construction de la réalité sociale, Paris: méridiens Klincksieck.

BODE, I. (2010) Extrait du livre Les services à la personne, sous la direction de Bernard Balzani, La documentation française.

CARADEC, V. (2001) Sociologie de la vieillesse et du vieillissement, Paris: Nathan.

CARADEC, V. (2003) La télévision, analyseur du vieillissement, Réseaux $\mathrm{N}^{\circ} 119$.

CHATTAT, R. (2004) L'invecchiamento: processi e strumenti di valutazione, Roma: Carocci.

CHOLAT, F. (2013) Impact chrono-géographique de la perte d'autonomie liée au vieillissement. Etude comparée de deux territoires alpins en France et en Italie, Mémoire de recherche de Master Sciences du Territoire spécialité Innovation et Territoire, sous la direction de Matteo Colleoni et Luc Gwiazdzinski, Grenoble, 2013, pp. 118.

COLLEONI, M. (2004) I tempi sociali. Teorie e strumenti di analisi, Roma: Carocci.

CUMMING, E. et W. HENRY (1961) Growing Old: The process of disengagement, New York: Basic books.

FONDATION DE FRANCE via TMO Régions, les solitudes en France, 2014.

FONTAINE, R. (2007) Psychologie du vieillissement, Ed Dunod.

GRAND, A., H. BOCQUET et S. ANDRIEU (2004) “Vieillesse et dépendance", Problèmes politiques et sociaux, N 903, La documentation française.

GUILLEMARD, A.-M. (2003) L'âge de l'emploi. Les sociétés à l'épreuve du vieillissement, Armand Colin.

GUINCHARD-KUNTSLER, P. (1999) Vieillir en France, rapport au premier ministre, SLSN.

GWIAZDZINSKI, L. et M. LYAZID (2006) "Vieillir à domicile”, Revue POUR N¹90, juin 2006, pp. 18-20.

HENRARD, J.-C. (2007) Répondre à la dépendance et aux handicapes: 5 ème risque ou 5 ème branche de sécurité sociale?, Gérontologie et société $N^{\circ} 123$, pp. 213-227.

HUGONOD, R. (2000) La vieillesse maltraitée, Dunod.

LORD, S. (2011) Le choix de vieillir à domicile: I'inévitable adaptation des modes de vie, Retraites et société $\mathrm{N}^{\circ} 60$.

NESTI, G. et al. (2003) Providing integrated health and social care for older persons in Italy, Padova Studio Come Srl, Roma.

PAILLAT, P. (1993) Vieillissement et vieillesse, Presses Universitaires Françaises, Etude.

SEGALEN, M. (1999) Le téléphone des familles, Réseaux N96.

VIRIOT-DURANDAL, J.-P. et al. (2012) Les défis territoriaux face au vieillissement, La documentation française.

$\S$
Una primera cronografía de la vida cotidiana del adulto mayor en los Alpes. Nuevas herramientas para favorecer la adaptación $>$ Luc Gwiazdzinski, Florent Cholat
Revista Márgenes N 19 Vol $13>$ Diciembre $2016>37$ Facultad de Arquitectura > Universidad de Valparaíso 